\title{
Hybrid Machine Learning Approach to Detect the Changes in SAR Images for Salvation of Spectral Constriction Problem
}

\author{
R. Dhaya, \\ Professor, \\ Department of Computer science and Engineering, \\ King Khalid University, \\ Kingdom of Saudi Arabia, \\ dhayavel2005@gmail.com
}

\begin{abstract}
For implementing change detection approaches in image processing domain, spectral limitations in remotely sensed images are remaining as an unresolved challenge. Recently, many algorithms have been developed to detect spectral, spatial, and temporal constraints to detect digital change from the synthetic aperture radar (SAR) images. The unsupervised method is used to detect the appropriate changes in the digital images, which are taken between two different consecutive periods at the same scene. Many of the algorithms are identifying the changes in the image by utilizing a similarity index-based approach. Therefore, it fails to detect the original changes in the images due to the recurring spectral effects. This necessitated the need to initiate more research for suppressing the spectral effects in the SAR images. This research article strongly believes that the unsupervised learning approach can solve the spectral issues to correct in the appropriate scene. The convolutional neural network has been implemented here to extract the image features and classification, which will be done through a SVM classifier to detect the changes in the remote sensing images. This fusion type algorithm provides better accuracy to detect the relevant changes between different temporal images. In the feature extraction, the semantic segmentation procedure will be performed to extract the flattened image features. Due to this procedure, the spectral problem in the image will be subsided successfully. The CNN is generating feature map information and trained by various spectral images in the dataset. The proposed hybrid technique has developed an unsupervised method to segment, train, and classify the given input images by using a pre-trained semantic segmentation approach. It demonstrates a high level of accuracy in identifying the changes in images.
\end{abstract}


Journal of Innovative Image Processing (JIIP) (2021)

Vol.03/ No. 02

Pages: 118-130

https://www.irojournals.com/iroiip/

DOI: https://doi.org/10.36548/jiip.2021.2.004

Keywords: Machine learning, Spectral constraints, SAR images

\section{INTRODUCTION}

The process of observing differences in the status of an object or phenomenon at different times involves detecting the changes [1]. One of the greatest problems is the Earth observation system, which has been extensively examined in recent decades. Multi-specific information on remote sensing (RS) can provide ample information for identifying the difference in land utilization and coverage, such as aerial and satellite photographs over the course of time within a given area. For various applications, such as urban planning, environmental monitoring, agriculture research, disaster assessment and map review, this is very important [2]. Figure 1 shows some sample SAR images.
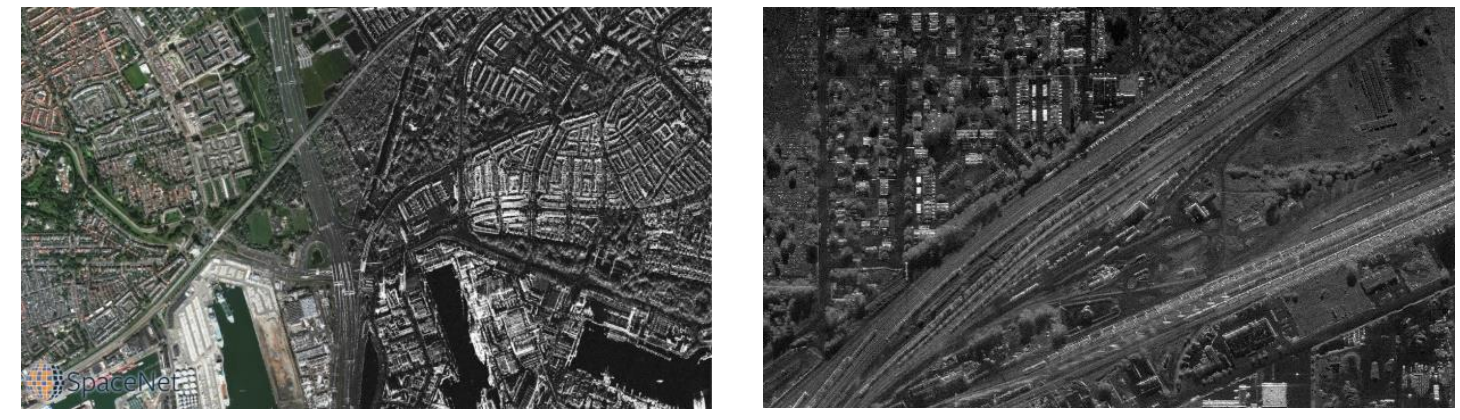

Figure 1 Sample SAR Images

The main concerns in the context of urban applications are changes in water systems, agricultural lands and buildings. However, various applications offer different areas of research in the urban scenario because of the diversity of the categories. Some scientists, for instance, focus part research on large-scale category changes [3, 4] and request intuitive changes to regional coverage $[5,6]$. Others also need to pay more attention to the changes in areas, not just to change areas [7] that need a more precise location and limits.

Naturally, the difference in the output requirements is considered as one of the problems for urban change detection technology. In the meantime, multi-source satellite transmitting sensors include various RS images, which can be used to detect changes, such as SAR images. Various RS pictures have different characteristics, such as SAR photos, multi-spectrum image 
Journal of Innovative Image Processing (JIIP) (2021)

Vol.03/ No. 02

Pages: 118-130

https://www.irojournals.com/iroiip/

DOI: https://doi.org/10.36548/jiip.2021.2.004

bands, and hyperspectral mixed imagery pixels. They also require a high level of universality and flexibility in the information extraction stage for change while providing adequate image resources [8]. Then, in view of multi-source and multi-target scenarios for urban change detection, it is difficult to decide which RS image data are more beneficial to balance the effective and exact findings of change detection [9]. This paper is aimed at creating and discussing the latest studies on urban change detection based on RS images. This research work encourages the researchers to concentrate on key technical points involved in specific data or application scenarios and explore technical optimization for detecting the changes.

\section{ORGANIZATION OF THE RESEARCH}

The structure of the research article is organized accordingly; some recent works on change detection methods in SAR images are included in Section 3. Section 4 provides an amendment to recent methods for detecting the future changes. Section 5 describes about various real-time tests. The conclusion and future tasks are covered in section 6 .

\section{PRELIMINARIES}

For the purpose of RS change detection tasks, image registration (e.g. co-registration), aligns multi-temporal images of same scene. Geographical registration is the most common registration strategy utilized for RS images by directly mapping multi-temporal images via automatic matching of control keys to the geographical coordinates of the digital raster RS images. However, data recording can be captured from different points of view, light environments, sensors and even different data models (e.g., digital elevation model). Invasion is therefore led to subsequent change detection (e.g. registration error change limit), which has evaluated the sensitiveness through misregistration [10].

In addition to the algorithm for the early match feature such as wavelet Transform and optical flow, the current research work promotes the creation of a deep network of key features or descriptors (e.g., contours, line-crossings, corner-bodies, etc.) based on the geographical registration and preliminary findings $[11,12]$. Dai et al seek correspondence between the features or perform 3D stereo modelling to solve difficulties in the remote sensing pictures. The 
Journal of Innovative Image Processing (JIIP) (2021)

Vol.03/ No. 02

Pages: 118-130

https://www.irojournals.com/iroiip/

DOI: https://doi.org/10.36548/jiip.2021.2.004

control points are used to guide image transformation mapping functions present in the image processing application. The sensitivity of the model has already been efficiently assessed [13].

As a result, the current research is advocating the use of deep networks to map key characteristics and/or designators (i.e. invariant features, contours, line intersections, corners etc.) based on preliminary geographical results, in addition to the early-feature matching algorithm like transform wavelet and optical flow [14]. They look for correspondence and similarity between the features or carry out 3D modelling on stereo to solve obstruction and multi-dimensional data problems. We must point out that, while the current registration algorithms perform well, registration is hard to achieve.

We noticed object detection and updating failures because of the misregistration observed from the above work. The current assessments of the change are mainly focused on the development of novel techniques for sensing the high-space image change [15]. The techniques under study are mainly traditional change detection methods, which can be summarised in the following groups:

\section{Fiscal Analysis:}

The changing map is interpreted manually by providing highly reliable and time consuming results based on expertise.

\section{Algebraic methods:}

The algebraic or cross-temporal data transformation such as image differentiation, image regression and image rationing can be used to obtain a map of change.

\section{Classification methods:}

Change is indicated by multiple (i.e. post-classification) classified chart comparisons or by qualified multi-period data classification classifiers (i.e.);

\section{Advanced modelling:}

Advanced models, such as li-reflection, spectral mixing of Strahler's and biophysical method for multiperodium data, are used for conversion of multifunctional reflection values into physical parameters or fractions [16]. 
Journal of Innovative Image Processing (JIIP) (2021)

Vol.03/ No. 02

Pages: 118-130

https://www.irojournals.com/iroiip/

DOI: https://doi.org/10.36548/jiip.2021.2.004

\section{METHODOLOGIES}

\subsection{Change Detection Framework}

In general, this is the technological process of detecting changes. Raw RS images are preprocessed with image recording to ensure the co-ordinate system is coherent. Following descriptions and attributes of the registered images, the multi-time information, named the fundamental features, can be obtained via the respective image extraction algorithms. It has color, texture, form, and space characteristics [17]. Then, feature changes are made to show the position and intensity of the information extracted about the change in order to identify whether it is refined or differentiated from the basic multi-temporal features.

\subsection{Standardization procedure}

The variation of illumination in the atmospheric condition, seasonal changes in the natures and sensor output at the time of capturing images should be homogenized for change detection [18]. Two methods that are utilized here are,

1. Geometric

2. Radiometric Correction

Through the standard registration, the former can align the dataset geometrically. The elimination of radiance and reflectance make differences for the digitalization process of the above said reasons. These heterogeneous data will be designed for feature galaxy conversion in order to accomplish change detection through standardization procedure [19].

\subsection{Mapping Techniques based Structure}

This type of framework structure is used to detect the changes in the dissimilar dataset. The Artificial Intelligence (AI) techniques are used to learn the feature mapping into another domain through a transformation that can perform with the heterogeneous dataset. The new neural network is based on a mapping function to detect multi-spatial resolution data and dissimilarity analysis through mapping transformation. The change detection is achieving in the SAR images through mapping transformation [20].

ISSN: 2582-4252 (online)

Submitted: 02.06.2021

Revised: 05.06.2021

Accepted: 16.06.2021

Published: 26.06.2021 
Journal of Innovative Image Processing (JIIP) (2021)

Vol.03/ No. 02

Pages: 118-130

https://www.irojournals.com/iroiip/

DOI: https://doi.org/10.36548/jiip.2021.2.004

\subsection{Dual Stream Procedure}

Here, the change detection is based on Siamese arrangement, transfer learning-based structure, and pole planning structure.

\section{Siamese Structure}

It gives two sub-nodes in the network for feature extractors, which are then used to transform into maps from two-period data. The deep features are getting training for testing for the neural networks. It will compare the weights of the sub-node and applied in the network structure by sharing the weights. The appropriate input data resulting in the trainable parameter which is used for feature extraction to detect the changes in the image [21].

\subsection{Proposed Framework}

The deep consideration of urban context, resources and environment, natural disasters, and astronomy effects (URNA) in our proposed network provides good sensitivity for changes in the detection. This URNA state is used to increasing the sensitivity rate and accuracy in the detection rate. Our proposed performs two-phase as $1 \& 2$. The feature extraction will be done through various dimension convolutional filters and the classifier can be done by support vector machine (SVM) approach [22, 23].

In the urban context, the expansion of the city and space management and building changes due to disaster will be accountable. Human and hydro drive environment changes and raises the water level in dam, river and extra will be accountable under the process of resource management. Landslide, damage in agriculture area due to nonstop rain is coming under natural disasters finally the planetary surfaces changes through astronomy [24, 25].

\subsubsection{Phase 1}

The proposed model includes a high-quality training set for the neural network in order to understand the spectral pattern from the SAR images. The manual annotation can be taken place to label the multi-period data. Input images are identified through a lot of convolutional filters and pooling layers.

The spectral constriction is the state of any SAR images. The spectral constriction problem is minimized through the consideration of URNA state in the SAR image change detection. The spectral features are extracted through the CNN approach in the deep learning 
Journal of Innovative Image Processing (JIIP) (2021)

Vol.03/ No. 02

Pages: 118-130

https://www.irojournals.com/iroiip/

DOI: https://doi.org/10.36548/jiip.2021.2.004

neural network after performing an appropriate image registration. This minimizing function has been performed after the minimization of squared error loss function and it is defined as,

$$
E s^{k}=\frac{1}{2} \sum_{i=1}^{n}\left(x_{i}^{k}-t_{i}^{k}\right)^{2}
$$

Where $x_{i}^{k}$ “i”"th objective sticky tag for the trial from 1 to $\mathrm{n}$.

The computation is very simple through convolution filters, which are used for registered images in order to implement change detection in the SAR images. The features extraction can be pushed up to the flattened procedure to train the model mathematically,

$$
\begin{gathered}
c_{i}=\rho\left(x_{i}\right) \\
x_{i}=W_{i} \cdot c_{i-1}+b_{i}
\end{gathered}
$$

The choosing of activation function is "ReLU", which provides better performance for the classifier. Figure shows our overall proposed framework.

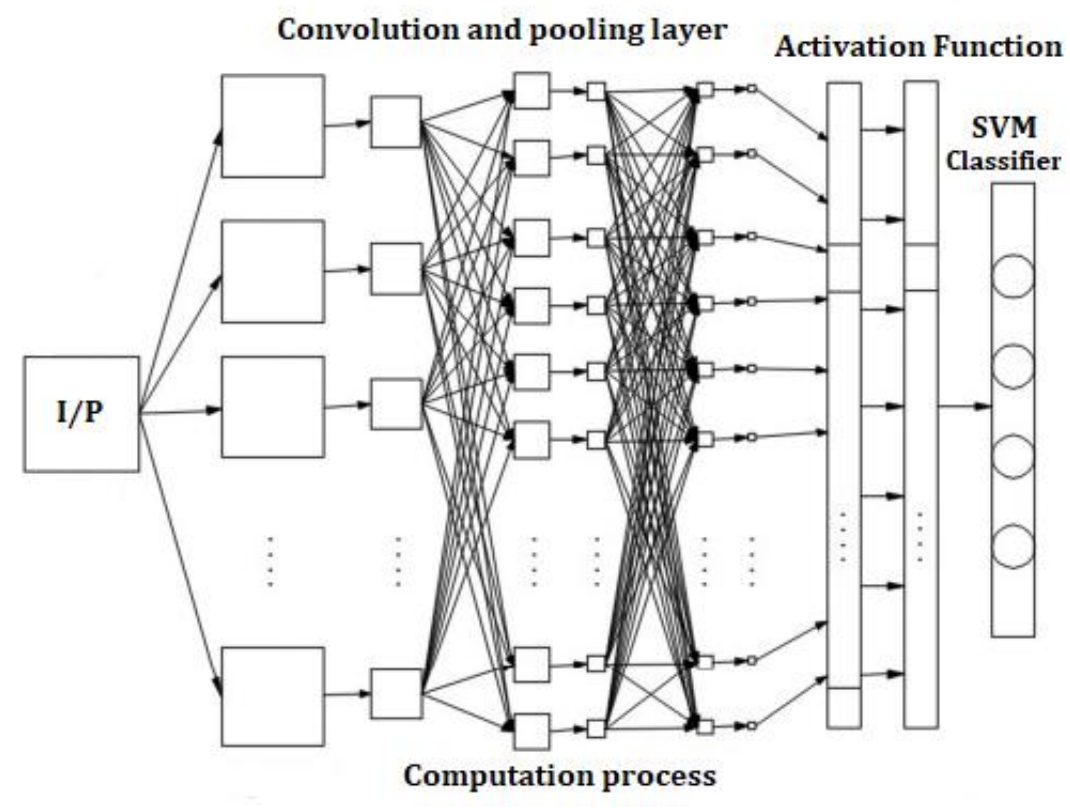

Figure 2 proposed frame work

\subsubsection{Phase 2}

The CNN architecture is combined with an SVM classifier to classify the detection and non-detection of changes in the SAR images over a certain period [26, 27]. The accurate 
Journal of Innovative Image Processing (JIIP) (2021)

Vol.03/ No. 02

Pages: $118-130$

https://www.irojournals.com/iroiip/

DOI: https://doi.org/10.36548/jiip.2021.2.004

unsupervised classification is performed through URNA state conditions for implementing a perfect identification of changes in the images obtained from the dataset. The mathematical derivation is expressed as;

$$
\left\{x_{i}, y_{i}\right\}_{i=1}^{n}
$$

The extracted features are trained in the neural network for solving the spectral constriction problem. The regression functions is a combination of activation function with weights and bias the node present in the network through the neural network, which is defined as,

$$
x=W \cdot c+b
$$

\section{RESULTS DISCUSSION}

Our proposed framework includes the annotated land change detection, which is combined with two dissimilar images by checking the ground truth label. The dataset is trained to get split for training and testing as $80 \%$ and $20 \%$ respectively to get the optimal value.
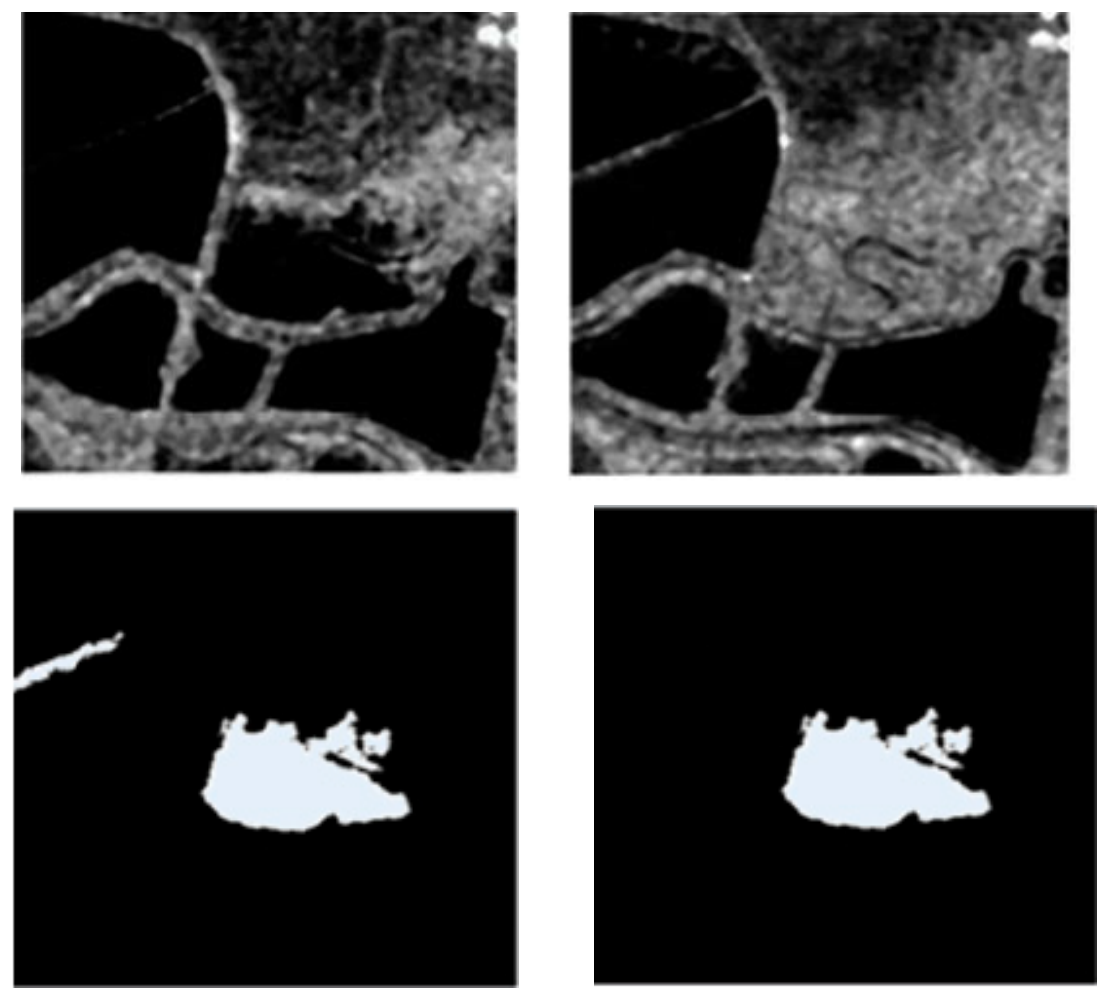

Figure 3 Obtained results Synthetic aperture radar (SAR) images 
Journal of Innovative Image Processing (JIIP) (2021)

Vol.03/ No. 02

Pages: 118-130

https://www.irojournals.com/iroiip/

DOI: https://doi.org/10.36548/jiip.2021.2.004

The test set for accuracy evaluation has been performed consecutively and iteratively.
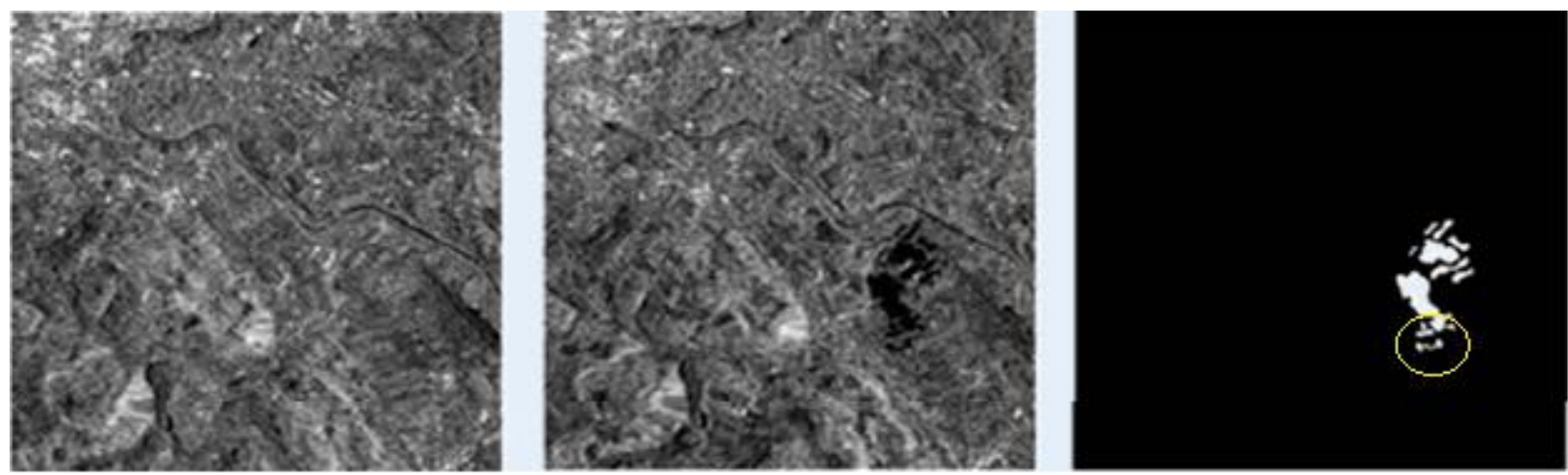

Figure 4 obtained results by proposed framework

The proposed model is well optimized according to learning conditions, which will minimize the loss function and solve the spectral constriction problem. The training process and test accuracy are monitored through the convergence of the model by validating its hyper parameters. Henceforth, a best performance has been achieved from our proposed model. Figures 3 and 4 show the results obtained by the existing methods and proposed method.

Table 1 Performance Metrics of Proposed Framework

\begin{tabular}{|c|c|c|c|c|c|c|}
\hline S.No & Methods & Recall & Precision & Accuracy & Sensitivity & $\begin{array}{c}\text { Recognition } \\
\text { error }(\%)\end{array}$ \\
\hline 1 & $\begin{array}{l}\text { Digital Image } \\
\text { processing }\end{array}$ & $86 \%$ & $79 \%$ & $81.5 \%$ & $80 \%$ & 0.071 \\
\hline 2 & $\begin{array}{l}\text { Mapping } \\
\text { technique }\end{array}$ & $80 \%$ & $77 \%$ & $80 \%$ & $75 \%$ & 0.175 \\
\hline 3 & Dual stream & $90 \%$ & $88 \%$ & $89 \%$ & $94 \%$ & 0.053 \\
\hline 4 & $\begin{array}{l}\text { Proposed hybrid } \\
\text { URNA based } \\
\text { algorithm }\end{array}$ & $92 \%$ & $89.5 \%$ & $92 \%$ & $97 \%$ & 0.008 \\
\hline
\end{tabular}

The proposed method is proving that, the superior change detection in SAR images is spotted by yellow circle as represented in the figure. The small changes can be determined by our proposed method. 


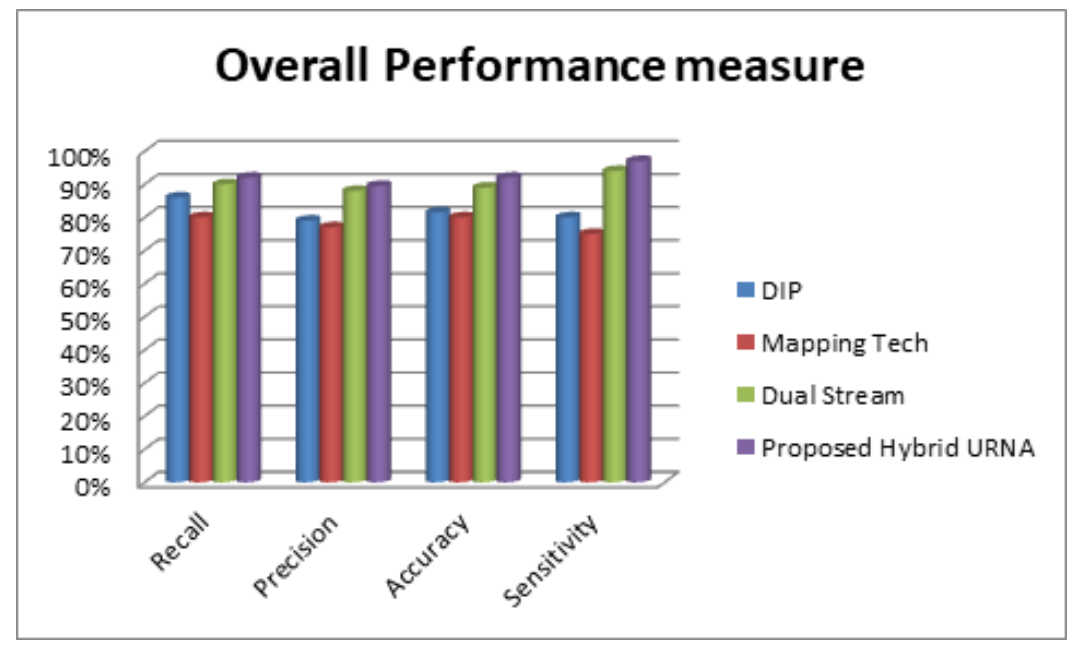

Figure 5 Overall Performance of the Proposed Method

The consideration of the URNA state by our proposed method has provided good accuracy results and a sensitive rate when compared to other existing methods. Table 1 shows the superiority of proposed method after calculating the model output. Figure 5 shows the graph on the overall performance of the proposed work and other existing methods.

\section{CONCLUSION}

Thus, the proposed work has achieved high sensitivity in detecting the changes present in SAR images. The spectral constriction problem has been minimized through pre-trained CNN with SVM classifier combination. The weights and bias of the neural network has been constructed through the training and testing process. The boundary effects removal is a good choice to get accurate results through the proposed algorithm. The change detection is improving by the URNA state of the proposed framework. In the future, the artificial intelligence method will be modified through multi retro input data category, accurate training set size with pre trained model and consideration on a high dimensional dataset present on the change map.

\section{REFERENCES}

[1] Sreedhar, Y., Najaraju, A., \& Krishna, G. M. (2016). An Appraisal of land use/land cover change scenari of Tummalapalle, cuddapah Region, India-A Remote Sensing, and GIS 
Journal of Innovative Image Processing (JIIP) (2021)

Vol.03/ No. 02

Pages: 118-130

https://www.irojournals.com/iroiip/

DOI: https://doi.org/10.36548/jiip.2021.2.004

Perspective. Advances in Remote Sensing, 5(4), 232-245. https://doi.org/10.4236/ars.2016. 54019.

[2] Vivekanadam, B. "Analysis of Recent Trend and Applications in Block Chain Technology." Journal of ISMAC 2, no. 04 (2020): 200-206.

[3] Koresh, H. James Deva, and Shanty Chacko. "Hybrid Speckle Reduction Filter for Corneal OCT Images." In International Conference on Image Processing and Capsule Networks, pp. 8799. Springer, Cham, 2020.

[4] Zhang, M.; Xu, G.; Chen, K.; Yan, M.; Sun, X. Triplet-Based Semantic Relation Learning for Aerial Remote Sensing Image Change Detection. IEEE Geosci. Remote Sens. Lett. 2019, 16, 266-270.

[5] Raj, Jennifer S. "Optimized Mobile Edge Computing Framework for IoT based Medical Sensor Network Nodes." Journal of Ubiquitous Computing and Communication Technologies (UCCT) 3, no. 01 (2021): 33-42.

[6] Ahmad, Misbah, Milind Ghawale, Sakshi Dubey, Ayushi Gupta, and Poonam Sonar. "GigaHertz: Gesture Sensing Using Microwave Radar and IR Sensor with Machine Learning Algorithms." In International Conference on Image Processing and Capsule Networks, pp. 422434. Springer, Cham, 2020.

[7] Zhuang, H.; Deng, K.; Fan, H.; Ma, S. A novel approach based on structural information for change detection in SAR images. Int. J. Remote Sens. 2018, 39, 2341-2365.

[8] Sivaganesan, D. "A Data Driven Trust Mechanism Based on Blockchain in IoT Sensor Networks for Detection and Mitigation of Attacks." Journal of trends in Computer Science and Smart technology (TCSST) 3, no. 01 (2021): 59-69.

[9] Liu, R.; Ku_er, M.; Persello, C. The Temporal Dynamics of Slums Employing a CNN-Based Change Detection Approach. Remote Sens. 2019, 11, 2844.

[10] Bindhu, V. "Constraints Mitigation in Cognitive Radio Networks Using Cloud Computing." Journal of trends in Computer Science and Smart technology (TCSST) 2, no. 01 (2020): 1-14

[11] De Jong, K.L.; Sergeevna Bosman, A. Unsupervised Change Detection in Satellite Images Using Convolutional Neural Networks. In Proceedings of the 2019 International Joint Conference on Neural Networks (IJCNN), Budapest, Hungary, 14-19 July 2019; pp. 1-8. 
Journal of Innovative Image Processing (JIIP) (2021)

Vol.03/ No. 02

Pages: 118-130

https://www.irojournals.com/iroiip/

DOI: https://doi.org/10.36548/jiip.2021.2.004

[12] Huang, Wenzhun, Shanwen Zhang, and Harry Haoxiang Wang. "Efficient GAN-Based Remote Sensing Image Change Detection Under Noise Conditions." In International Conference on Image Processing and Capsule Networks, pp. 1-8. Springer, Cham, 2020.

[13] Dai, X.; Khorram, S. The e_ects of image misregistration on the accuracy of remotely sensed change detection. IEEE Trans. Geosci. Remote Sens. 1998, 36, 1566-1577

[14] Mugunthan, S. R., and T. Vijayakumar. "Design of Improved Version of Sigmoidal Function with Biases for Classification Task in ELM Domain." Journal of Soft Computing Paradigm (JSCP) 3, no. 02 (2021): 70-82.

[15] Zhao, W.; Mou, L.; Chen, J.; Bo, Y.; Emery, W.J. Incorporating Metric Learning and Adversarial Network for Seasonal Invariant Change Detection. IEEE Trans. Geos

[16] Adam, Edriss Eisa Babikir. "Evaluation of Fingerprint Liveness Detection by Machine Learning Approach-A Systematic View." Journal of ISMAC 3, no. 01 (2021): 16-30.

[17] Cui, B.; Zhang, Y.; Yan, L.;Wei, J.; Huang, Q. A SAR change detection method based on the consistency of single-pixel difference and neighbourhood difference. Remote Sens. Lett. 2019, 10, 488-495.ci. Remote Sens. 2020, 58, 2720-2731.

[18] Hamdan, Yasir Babiker. "Faultless Decision Making for False Information in Online: A Systematic Approach." Journal of Soft Computing Paradigm (JSCP) 2, no. 04 (2020): 226-235

[19] Wu, C.; Du, B.; Zhang, L. Hyperspectral anomalous change detection based on joint sparse representation. ISPRS J. Photogramm. Remote Sens. 2018, 146, 137-150.

[20] Shakya, Subarna. "Process mining error detection for securing the IoT system." Journal of ISMAC 2, no. 03 (2020): 147-153.

[21] Zhang, X.; Shi, W.; Lv, Z.; Peng, F. Land Cover Change Detection from High-Resolution Remote Sensing Imagery Using Multitemporal Deep Feature Collaborative Learning and a Semisupervised Chan-Vese Model. Remote Sens. 2019, 11, 2787.

[22] Adam, Edriss Eisa Babikir. "Survey on Medical Imaging of Electrical Impedance Tomography (EIT) by Variable Current Pattern Methods." Journal of ISMAC 3, no. 02 (2021): 82-95.

[23] Wan, L.; Xiang, Y.; You, H. An Object-Based Hierarchical Compound Classification Method for Change Detection in Heterogeneous Optical and SAR Images. IEEE Trans. Geosci. Remote Sens. 2019, 57, 9941-9959. 
Journal of Innovative Image Processing (JIIP) (2021)

Vol.03/ No. 02

Pages: 118-130

https://www.irojournals.com/iroiip/

DOI: https://doi.org/10.36548/jiip.2021.2.004

[24] Kohli, Himani, Parth Sagar, Atul Kumar Srivastava, Anuj Rani, and Manoj Kumar. "A Machine Learning Approach to Detect Image Blurring." In Computational Vision and BioInspired Computing, pp. 315-325. Springer, Singapore, 2021.

[25] Ranganathan, G. "Real time anomaly detection techniques using pyspark frame work." Journal of Artificial Intelligence 2, no. 01 (2020): 20-30.

[26] Kwan, C.; Ayhan, B.; Larkin, J.; Kwan, L.; Bernabé, S.; Plaza, A. Performance of Change Detection Algorithms Using Heterogeneous Images and Extended Multi-attribute Profiles (EMAPs). Remote Sens. 2019, 11, 2377.

[27] Devakumari, D., and V. Punithavathi. "Noise Removal in Breast Cancer Using Hybrid Denoising Filter for Mammogram Images." In International Conference On Computational Vision and Bio Inspired Computing, pp. 109-119. Springer, Cham, 2019.

\section{Author's Biography}

R. Dhaya is currently a Professor, in the Department of Computer science and Engineering at King Khalid University, in Kingdom of Saudi Arabia. His major area of research includes Image and Video Processing Algorithms, Computer Vision, Motion Analysis, Stereo Vision, Object Recognition, computer graphics, photo interpretation, image retrieval, Embedded Image Processing and Real-time image and video processing applications.

ISSN: 2582-4252 (online) 